\title{
Self-Integrating and Self-Improving Systems Must Be Socially Sensitive
}

\author{
Peter R. Lewis \\ Aston Lab for Intelligent Collectives Engineering (ALICE) \\ Aston University, Birmingham,UK p.lewis@aston.ac.uk
}

In this talk I will discuss two areas of recent research relevant to socially-inspired technical systems that engage in self-integration and self-improvement [1]. The first of these is socially-sensitive systems design [2]. This is a new way thinking about the design of complex systems that explicitly considers their runtime awareness of, and behaviour based on, those social factors with the potential to affect their operation. I will present a conceptual framework for sociallysensitive systems design [2], that draws on research in selforganisation and sociology, and comprises aspects related to social values, social relations and social organisation. A key benefit for such systems will be derived from the notion of better positioning through increased social potential, enabling entities within the system to achieve goals (e.g., self-integration and collective adaptation) quickly. This is achieved through the establishment of shared social values, norms, rules and networks, that are agreed upon, learnt quickly, and sustained across a population and over time.

Secondly, I will highlight is role and importance of social learning in systems where fast integration is needed. Using examples from animal behaviour, I will explore the role of social and asocial learning in self-integration and self-improvement, highlighting that the two can sometimes be in tension with each other. Specifically, social learning can play two important roles in self-integrating, selfimproving systems, which may be complementary or conflictual. First, social learning can make learning faster [3]. In a challenging environment, good behaviours may be difficult to find by an individual engaging in exploratory learning, yet once found, it is beneficial to share this information quickly around the group, to others faced with the same problem. Second, in a co-adaptive setting, as we may be faced with in self-integrating systems, fitness is a function of the individual and the group's behaviour. Here it matters less precisely what the behaviour is in an absolute sense, and more whether the group have learned to behave in the same way as each other (e.g., learning to use a common API). An example of this is "traditions" within meerkat societies, which determine factors such as their waking times [4], and are learnt and passed down through generations.

A common assumption about animal learning is that learning by copying is always beneficial. However, this is known to be flawed [3], as social learners typically only have higher fitness than asocial learners when copying is infrequent, and asocial exploratory learning is the dominant form of learning in a population. Indeed, Coolen and Laland note that "individuals appear to switch between reliance on social and asocial sources of information in a flexible and facultative manner" [5, p369], and argue that models of learning should incorporate both social and asocial learning in a flexible way. This would appear to be a promising approach for self-integrating and self-improving systems, where behavioural learning is required to i) be fast, ii) align with the learning of others, and iii) continue to find new ways to improve in a dynamic environment.

For the reasons given above, it will be important to integrate both socially-sensitive systems design and social learning into self-integrating and self-improving systems. When doing so, there are a number of new issues and questions that arise. Therefore, I will conclude by proposing directions for future research, in particular highlighting one such issue demonstrated by some of our recent results.

Specifically, a key feature of systems that improve through independent asocial learning is exploration. Therefore, social organisations will need to be tolerant of exploratory behaviour, even though this may violate what is deemed acceptable behaviour by the group. A sociallysensitive self-improving system will need to be aware, either implicitly or explicitly, of the importance of exploratory behaviour, and act accordingly. However, we recently showed [6] that in institutions (social organisations governed by mutually agreed rules) there is an interplay between the rules and the scope of agents' learning. The way in which agents learn has an impact on the existence and sustainability of the institution, while the properties of the institution can either tolerate or inhibit different forms of learning, and thus self-integration and/or self-improvement.

\section{References}

[1] K. Bellman, S. Tomforde, and R. P. Würtz, "Interwoven systems: Self-improving systems integration," in IEEE Eighth International Conference on Self-Adaptive and Self-Organizing Systems Workshops (SASOW), 2014, pp. 123-127.

[2] K. Bellman, J. Botev, H. Hildmann, P. R. Lewis, S. Marsh, J. Pitt, I. Scholtes, and S. Tomforde, "Socially-sensitive systems design," IEEE Technology and Society Magazine, 2017, to appear.

[3] K. N. Laland, "Social learning strategies," Learning \& Behavior, vol. 32, pp. 4-14, 2004.

[4] A. Thornton, Samson, J., and C.-B. T., "Multi-generational persistence of traditions in neighbouring meerkat groups." 2010.

[5] I. Coolen and K. N. Laland, "Trade-offs in the adaptive use of social and asocial learning," Advances in the Study of Behavior, vol. 35, pp. 333-379, 2005.

[6] P. R. Lewis and A. Ekárt, "Social and asocial learning in collective action problems: The rise and fall of socially-beneficial behaviour," in Proceedings of the IEEE 2nd International Workshops on Foundations and Applications of Self* Systems, FAS-W 2017, to appear. 